\title{
L-carnitine Supplementation Enhances Nuclear and Cytoplasmic Maturation Rates of Sheep Oocytes In Vitro
}

\author{
Z. W. Bhakty ${ }^{a}$, E. M. Kaiin ${ }^{b}$, N. W. K. Karja ${ }^{a, c}$, \& M. A. Setiadi ${ }^{a, c, *}$ \\ aBiology of Reproduction, Graduate School of IPB University \\ ${ }^{b}$ Research Center for Biotechnology, Indonesian Institute of Sciences (LIPI), Indonesia \\ 'Division of Reproduction and Obstetric, Department of Veterinary Clinic, Reproduction, and Pathology, \\ Faculty of Veterinary Medicine, IPB University \\ Jalan Agatis, Kampus IPB Dramaga Bogor 16680, Indonesia \\ ${ }^{*}$ Corresponding author: setiadi03@yahoo.com \\ (Received 14-07-2020; Revised 09-11-2020; Accepted 16-11-2020)
}

\begin{abstract}
The aim of the present study was to determine the effectiveness of 1-carnitine (LC) supplementation on nuclear and cytoplasmic maturation rates of sheep oocytes. In experiment 1 , oocytes were maturated for 24 hours in tissue culture medium 199 supplemented with LC at doses of $0.3 \mathrm{mg} / \mathrm{mL}, 0.6 \mathrm{mg} / \mathrm{mL}$, and $0.9 \mathrm{mg} / \mathrm{mL}$. In experiment 2 , oocytes were maturated and fertilized in a media supplemented with LC at a dose of $0.3 \mathrm{mg} / \mathrm{mL}$ and incubated with $5 \times 10^{6} \mathrm{sperm} / \mathrm{mL}$ for 12 hours. The treatment group consisted of LC supplementation only in maturation medium $\left(P_{1}\right)$, only in fertilization medium $\left(\mathbf{P}_{2}\right)$, and in both maturation and fertilization media $\left(\mathrm{P}_{3}\right)$. In experiment 3 , sperm motility patterns were assessed using CASA after being exposed to fertilization medium supplemented with LC at a dose of $0.3 \mathrm{mg} / \mathrm{mL}$ for 0 and 3 hours. Our results showed that supplementation of LC at a dose of $0.3 \mathrm{mg} / \mathrm{mL}$ significantly $(\mathrm{p}<0.05)$ increased the percentage of oocytes reaching metaphase II $(86.7 \pm 4.1 \%)$ compared to those supplemented with $\mathrm{LA}$ at doses of $0,0.6$, and $0.9 \mathrm{mg} / \mathrm{mL}(73.6 \pm 1.2 \%, 81.4 \pm 1.3 \%$, and $70.5 \pm 1.6 \%$, respectively). The LC treatment in the fertilization medium only did not influence the number of two pronuclear formations $(62.1 \pm 2.5 \%)$ compared to supplementation either in the maturation medium only $(72.0 \pm 4.7 \%)$ or a combination of both in maturation and fertilization media $(68.2 \pm 2.7 \%)(p<0.05)$. Further results after 3 hours of incubation compared to the control group showed the total motility $(24.8 \pm 2.04 \%$ vs. $17.49 \pm 2.37 \%)$, progressive motility $(14.17 \pm 2.03 \%$ vs. $6.49 \pm 1.64 \%)$, and curvilinear velocity (VCL) $(119.70 \pm 3.73 \%$ vs. $71.15 \pm 10.59 \%)(p<0.05)$ were increased in the fertilization medium containing LC but it did not improve the fertilization rate. It is concluded that supplementation of $\mathrm{LC}$ at a dose of $0.3 \mathrm{mg} / \mathrm{mL}$ in the maturation medium only could better improve the nuclear and cytoplasmic maturation rates of sheep oocytes.
\end{abstract}

Keywords: fertilization; l-carnitine; maturation; oocyte; sheep

\section{INTRODUCTION}

The systems of in vitro embryo production (IVEP) have been widely used for livestock to improve quality production with the various benefits provided (Egashira et al., 2019). However, IVEP in sheep is quite low compared to the other livestock species such as cattle or pigs (Paramio \& Izquierdo, 2014). In general, the low rate of IVEP in livestock is caused by the low rate of oocyte nuclear and cytoplasmic maturation (El-Raey \& Nagai, 2014). The rate of nuclear maturation usually is assessed based on the number of oocytes reaching the stage of metaphase II. Meanwhile, one of the criteria that is generally used to assess cytoplasmic maturation is the number of oocytes forming pronuclear after fertilization (Curnow et al., 2010). Therefore, synchronization between nuclear and cytoplasmic maturation is required to support fertilization and subsequent development after fertilization (El-Raey \& Nagai, 2014).

The incidences of low rates of nuclear maturation and fertilization occur due to the negative effects of reactive oxygen species (ROS) (Lopes et al., 2010), which is produced during in vitro culture (Troung \& Gardner, 2017). It is reported that increased levels of ROS during the process of in vitro maturation (IVM) can trigger oxidative stress in the oocytes (Lobo et al., 2010). Oxidative stress can cause damages to carbohydrates, lipids, proteins (Wang et al., 2017), and DNA in the oocytes (Lobo et al., 2010) that eventually decrease the oocyte competence. In addition, the increased levels of ROS during the process of in vitro fertilization (IVF) can result in the failure of fertilization (Opuwari \& Henkel, 2016). These conditions can be minimized by the supplementation of an antioxidant in the media (Shafiei et al., 2020). One of the potential antioxidants, which has an important role either as scavenging of ROS or as an agent to support nuclear maturation and fertilization, is 1-carnitine (Phongmitr et al., 2013).

L-carnitine is a water-soluble, small, and highly polar molecule that is crucial for lipid metabolism (Jiang et al., 2020). Furthermore, many researchers (Phongmitr et al., 2013; Fathi \& El-Shahat, 2017) have been associated 
the useful effects of LC with its roles as an antioxidant (ROS scavenger), an enhancer of lipid metabolism via $\beta$-oxidation, and in increasing glutathione (GSH) levels. As an antioxidant, LC has an activity to protect the damages of mitochondrial membrane and DNA induced by ROS and inhibited cell apoptosis (Wu et al., 2011). L-carnitine has a higher antioxidant activity than the other antioxidants such as tocopherol and Trolox (Kelek et al., 2019). Furthermore, LC plays a crucial role in the metabolism of lipid by transporting long-chain fatty acids from the cytosol into the mitochondria for further used in the $\beta$-oxidation reactions to produce the adenosine triphosphate (ATP), which is required for the metabolism of an oocyte (Phongmitr et al., 2013; Agarwal et al., 2018). The strong source of ATP in the cell is the metabolism of fatty acids via $\beta$-oxidation that improve the processes of oocytes maturation and development after fertilization (Dunning \& Robker, 2017).

In addition, the benefit of LC could increase the activity and levels of glutathione (GSH) (Sovergino et al., 2017). GSH improves the development competence of mammalian oocytes at the cytoplasmic level (Curnow et al., 2010). Cytoplasmic maturation is required during early fertilization and embryogenesis after fertilization (Reader et al., 2017). Dokmeci (2005) showed the beneficial effect of LC on spermatozoa in IVEP of mice by improving motility and quality of chromatin. However, information concerning the effects of LC during the maturation and fertilization of sheep oocytes in vitro is still limited. Therefore, this study was conducted to examine the effectiveness of LC supplementation on nuclear and cytoplasmic maturation of sheep oocytes.

\section{MATERIALS AND METHODS}

\section{Assessment of Functional Activity of L-carnitine on Nuclear Maturation}

Oocytes collection and maturation in vitro. The maturation process was carried out according to Riyuska et al. (2019) with minor modifications. Sheep ovaries were collected from a local slaughterhouse and then carried to the laboratory in $0.9 \% \mathrm{NaCl}$ with $0.1 \mathrm{mg} / \mathrm{mL}$ of streptomycin (MEIJI, Indonesia) and $100 \mathrm{IU} / \mathrm{mL}$ of penicillin (MEIJI, Indonesia). The oocytes were collected by repeatedly slicing the ovaries with a scalpel blade (slicing technique) in a sterile petri dish containing phosphatebuffered saline (PBS), supplemented with $0.1 \mathrm{mg} / \mathrm{mL}$ of streptomycin (Sigma-Aldrich. Inc, S9137. USA), 100 IU/ $\mathrm{mL}$ of penicillin (Sigma-Aldrich. Inc, P-4687. USA), and $0.3 \%$ bovine serum albumin (BSA) (Sigma-Aldrich. Inc, A7030. USA). Only oocytes surrounded by more than three layers of cumulus cells and with homogeneous cytoplasm were used in this experiment.

Selected Cumulus oocyte complex (COCs) were then washed three times in the maturation medium. The oocytes were matured in $100 \mu \mathrm{L}$ drop of maturation medium for 10-15 oocytes covered by mineral oil. The composition of the maturation medium is tissue culture medium 199 (TCM-199) (Sigma-Aldrich, St. Loius, M4530, USA) supplemented with BSA at a dose of $0.3 \%$, human chorionic gonadotrophin (hCG) (Intervet Boxmeer-Holland) at a dose of $10 \mathrm{IU} / \mathrm{mL}$, gentamycin
(Sigma-Aldrich, G1264, USA) at a dose of $50 \mu \mathrm{g} / \mathrm{mL}$, and follicle stimulating hormone (FSH) (Vetoquinol N,-A Inc, Canada) at a dose of $10 \mathrm{IU} / \mathrm{mL}$. The oocytes were maturated in 4 different concentrations of LC (C0158, Sigma-Aldrich, St. Louis, USA) treatments, namely 0 (control), 0.3, 0.6, and $0.9 \mathrm{mg} / \mathrm{mL}$, respectively. The oocytes were maturated under $5 \% \mathrm{CO}_{2}$ at $38.5^{\circ} \mathrm{C}$ in humidified air for 24 hours.

Assessment of nuclear maturation. The oocytes were separated from their cumulus cells using the $0.25 \%$ hyaluronidase enzyme (Sigma-Aldrich, St. Louis, H3506, USA) in a sterile petri dish and repeated pipetting with a fit pipette adjusting to the measure of the oocytes. The oocytes were completely denuded, then washed within PBS $+0.3 \%$ BSA, and placed on a glass slide under coverslips. The slide was placed in a fixative solution mixture of acetic acid (K45626263 420, Merck, Germany) and absolute ethanol (K44151883 303, Merck, Germany) in the ratio of 1:3 for $48-72$ hours. Then, the slide was stained using $2 \%$ aceto-orcein (Sigma-Aldirch, St. Louis, O7380, USA) and examined under a phase-contrast microscope (Olympus IX 70, Japan). The oocytes were classified based on the changes of chromosome configuration and nuclear membrane, namely germinal vesicle $(\mathrm{GV})$, germinal vesicle break down (GVBD), metaphase I (MI), anaphase and telophase I (A/TI), and metaphase II (MII) (Figure 1). The germinal vesicle was characterized by condensed or slightly diffused chromatin, whereas GVBD stage was characterized by diffused chromatin in which the nuclear membrane was fragmented before rapidly disappearing to leave only small sacs with double walls. Metaphase I (MI) stage was characterized by strongly condensed chromatin that formed an irregular network of individual bivalents (prometaphase) or a metaphase plate without the polar body. Oocytes classified as Metaphase II (MII) stage was characterized by the presence of either a polar body or two chromatin masses (Shirazi \& Sadeghi 2007).

\section{Assessment of Functional Activity of L-carnitine on Cytoplasmic Maturation}

In vitro fertilization. L-carnitine was supplemented into the medium either in IVM or IVF and a combination using the best concentration obtained from the results of previous experiments $(0.3 \mathrm{mg} / \mathrm{mL})$. The LC treatments were divided into four groups, namely without LC supplementation either in IVM or IVF medium (P0) as controls, LC supplementation only in IVM medium (P1), or in IVF medium (P2), and supplementation both in the IVM and IVF media (P3). Frozen semen of sheep obtained from an artificial insemination center, Lembang, Bandung, West Java, Indonesia, was used for in vitro fertilization. The fertilization process was done according to Riyuska et al. (2019) with minor modifications. The frozen semen was thawed in a water bath at $37^{\circ} \mathrm{C}$ for 30 seconds and then diluted with IVF medium (Suzuki et al., 2000) of $0.5 \mathrm{mM} \mathrm{MgSO}_{4}$ containing $12 \mathrm{mK}$ $\mathrm{KCl}, 0.5 \mathrm{mM} \mathrm{NaH} \mathrm{PO}_{4}, 90 \mathrm{mM} \mathrm{NaCl}, 25 \mathrm{mM} \mathrm{NaHCO}{ }_{3}$ $10 \mathrm{mM}$ sodium lactate, $5 \mathrm{mM}$ caffeine, $3 \mathrm{mg} / \mathrm{mL}$ BSA, and $50 \mu \mathrm{g} / \mathrm{mL}$ gentamicin. The diluted semen was then centrifuged at $500 \times \mathrm{g}$ for 5 minutes. After centrifuga- 


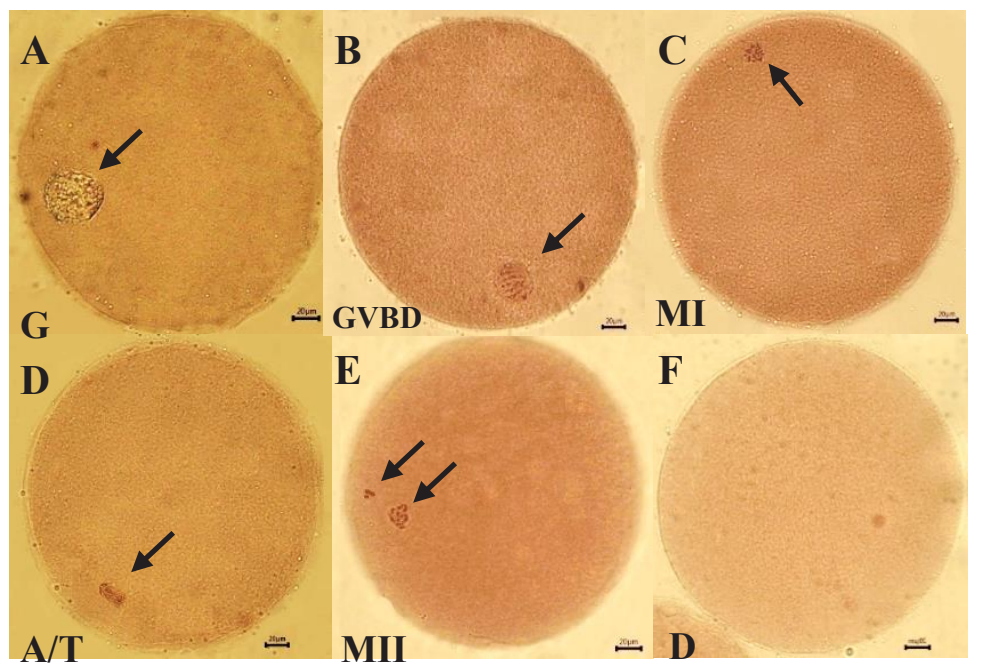

Figure 1. Nuclear maturation stage of oocytes in various l-carnitine concentrations in the maturation medium. $\mathrm{A}=$ germinal vesicle; $\mathrm{B}=$ germinal vesicle breakdown; $\mathrm{C}=$ metaphase $\mathrm{I} ; \mathrm{D}=$ anaphase/telophase, $\mathrm{E}=$ metaphase II; $\mathrm{F}=$ degeneration; $\mathrm{GV}=$ germinal vesicle; $\mathrm{GVBD}=$ germinal vesicle break down; $\mathrm{MI}=$ metaphase $\mathrm{I} ; \mathrm{A} / \mathrm{TI}=$ anaphase and telophase I; MII= metaphase II; $\mathrm{D}=$ degeneration.

tion, the supernatant was discarded, then the pellet was diluted with the IVF medium to a final concentration of $5 \times 10^{6}$ sperm $/ \mathrm{mL}$ and put in a droplet $(100 \mu \mathrm{L})$. After the maturation process, the oocytes were washed 3 times using the fertilization medium without sperm, then incubated into the droplet of sperm $(100 \mu \mathrm{L})$ in $5 \% \mathrm{CO}_{2}$, at $38.5^{\circ} \mathrm{C}$ in humidified air for 12 hours.

Assessment of cytoplasmic maturation. The assessment of the cytoplasmic maturation was determined by the formation of pronuclear (El-Raey \& Nagai, 2014). The normal fertilization was classified by the formation of two pronuclear, while the oocyte having more than two pronuclear was classified as polyspermy. The cytoplasmic maturation rate was evaluated with $2 \%$ aceto-orcein (Sigma-Aldirch, St. Louis, O7380, USA) after 12 hours of fertilization process and examined under a phase-contrast microscope (Olympus IX 70, Japan).

Assessment of sperm motility. In this experiment, LC was supplemented into the fertilization medium with the best concentration in the previous experiment $(0.3$ $\mathrm{mg} / \mathrm{mL}$ ). Samples of each treatment were assessed for the pattern of sperm motility using the CASA system (Sperm Vision ${ }^{\mathrm{TM}}$ 3.7 Minitube, Germany). Parameters measured were percentages of total motility, progressive motility, the amplitude of lateral head displacement (ALH), linearity (LIN), and curvilinear velocity (VCL). Sperm sample for analysis was obtained from sperm sample derived from IVF process according to the treatment. Analysis of the pattern of sperm motility was conducted 0 and 3 hours after the incubation period to record the pattern of motility as an indicator of the capacitation process to improve fertilization rate.

\section{Statistical Analysis}

Nuclear maturation and fertilization rates were analyzed statistically with One-Way Analysis of
Variance (ANOVA). The patterns of sperm motility were assessed using Two-Way ANOVA. Differences at a probability value of $\mathrm{p}<0.05$ were considered to be statistically significant.

\section{RESULTS}

\section{Nuclear Maturation Rate}

The percentages of oocytes in MII stage in the maturation medium supplemented with various concentrations of LC $(0,0.3 \mathrm{mg} / \mathrm{mL}, 0.6 \mathrm{mg} / \mathrm{mL}$, and $0.9 \mathrm{mg} / \mathrm{mL})$ were $73.6 \pm 1.2 \%, 86.7 \pm 4.1 \%, 81.4 \pm 1.3 \%$, and $70.5 \pm 1.6 \%$, respectively (Table 1 ). The results of our study revealed that supplementation of LC at a dose of $0.3 \mathrm{mg} / \mathrm{mL}$ was the best result in improving the nuclear maturation compared to the other treatments and control $(p>0.05)$.

\section{Cytoplasmic Maturation and Pattern of Sperm Motility Rate}

The success of cytoplasmic maturation in vitro could be assessed based on the formation of pronuclear (El-Raey \& Nagai, 2014). The formation of two pronuclear was categorized as normal fertilization. Meanwhile, if the oocyte had more than two pronuclear was categorized as polyspermy (Figure 2). The result of the experiment revealed that the formation of two pronuclear was higher with the supplementations of LC in the maturation medium (P1) $(72.0 \pm 4.7 \%)$ and in the combination of maturation and fertilization media (P3) $(68.2 \pm 2.7 \%)$ compared to the supplementation of LC only in the fertilization medium (P2) $(60.5 \pm 5.1 \%)$ and control without LC supplementation $(62.1 \pm 2.5 \%)$ $(p<0.05)$. Supplementation of LC only in the fertilization medium did not improve the normal fertilization. In addition, the results of the experiment showed the increasing number of polyspermy $(14.8 \pm 2.3 \%)(\mathrm{p}<0.05)$ in the LC supplementation in the fertilization medium (P2) 
Table 1. Nuclear maturation rate of sheep oocytes in different concentrations of L-carnitine in the maturation medium

\begin{tabular}{ccccrccc}
\hline \multirow{2}{*}{$\begin{array}{c}\text { Concentration of } \\
\text { LC }(\mathrm{mg} / \mathrm{mL})\end{array}$} & \multirow{2}{*}{ No of oocytes } & \multicolumn{5}{c}{ Nuclear maturation rate $(\%$, mean \pm SD) } \\
\cline { 3 - 8 } & & GV & GVBD & \multicolumn{1}{c}{ MI } & \multicolumn{1}{c}{ A/T } & MII & D \\
\hline 0 & 110 & $1.8 \pm 0.5$ & $7.3 \pm 4.0$ & $12.7 \pm 4.7$ & $0.0 \pm 0.0^{\mathrm{a}}$ & $73.6 \pm 1.2^{\mathrm{a}}$ & $4.5 \pm 1.5^{\mathrm{a}}$ \\
0.3 & 113 & $0.9 \pm 0.0$ & $3.5 \pm 2.6$ & $8.0 \pm 3.3$ & $0.9 \pm 0.0^{\mathrm{a}}$ & $86.7 \pm 4.1^{\mathrm{b}}$ & $0.0 \pm 0.0^{\mathrm{b}}$ \\
0.6 & 102 & $2.9 \pm 5.9$ & $2.9 \pm 3.7$ & $12.7 \pm 5.5$ & $0.0 \pm 0.0^{\mathrm{a}}$ & $81.4 \pm 1.3^{\mathrm{c}}$ & $0.0 \pm 0.0^{\mathrm{b}}$ \\
0.9 & 105 & $2.9 \pm 0.6$ & $5.7 \pm 5.4$ & $13.3 \pm 3.4$ & $7.6 \pm 6.3^{\mathrm{b}}$ & $70.5 \pm 1.6^{\mathrm{d}}$ & $0.0 \pm 0.0^{\mathrm{b}}$ \\
\hline
\end{tabular}

Note: $\mathrm{LC}=\mathrm{L}$-carnitine; $\mathrm{GV}=$ germinal vesicle; $\mathrm{GVBD}=$ germinal vesicle break down; $\mathrm{MI}=$ metaphase $\mathrm{I} ; \mathrm{A} / \mathrm{T}=$ anaphase and telophase; $\mathrm{MII}=$ metaphase II; $\mathrm{D}=$ degeneration. Means in the same columns with different superscripts differ significantly $(\mathrm{p}<0.05)$.

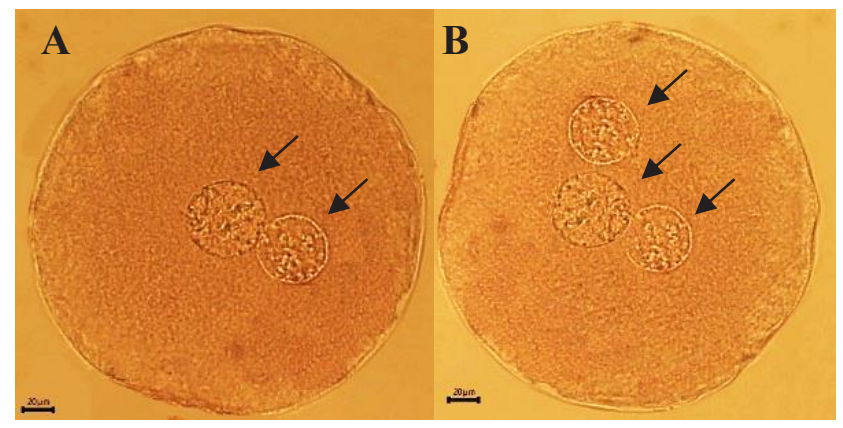

Figure 2. Pronuclear development of sheep oocyte after fertilization in different l-carnitine supplementations. $\mathrm{A}=2$ pronuclear; $\mathrm{B}=>2$ pronuclear.

only compared to the control and the other treatments (Table 2).

The patterns of sperm motility (total motility, progressive motility, and curvilinear velocity (VCL)) in the medium supplemented with LC at a dose of $0.3 \mathrm{mg} / \mathrm{mL}$ revealed the significantly higher at 0 hour of incubation $(55.12 \pm 4.13 \%, 40.66 \pm 7.92 \%$, and $129.53 \pm 11.50 \%$, respectively) and after 3 hours of incubation $(24.8 \pm 2.04 \%$, $14.17 \pm 2.03 \%$, and $119.70 \pm 3.73 \%$, respectively) compared to the controls at 0 hour of incubation $(42.05 \pm 1.79 \%$, $25.21 \pm 6.74 \%$, and $1.73 \pm 1.56 \%$, respectively) and after 3 hours of incubation $(17.49 \pm 2.37 \%, 6.49 \pm 1.64 \%$, and $71.15 \pm 10.59 \%$, respectively) $(\mathrm{p}<0.05) \quad$ (Figure $3)$. However, the parameters of linearity (LIN) and amplitude of lateral head displacement (ALH) of the sperms were not significantly different in the medium supplemented with $0.3 \mathrm{mg} / \mathrm{mL}$ LC either at 0 hour $(0.54 \pm 0.04 \%$ and $4.80 \pm 0.62 \%)$ and after 3 hours of incubation $(0.61 \pm 0.15 \%$ and $3.80 \pm 0.95 \%)$ compared with control without LC treatment at 0 hour of incubation
$(0.68 \pm 0.23 \%$ and $3.93 \pm 1.70 \%)$ and after 3 hours of incubation $(0.69 \pm 0.16 \%$ and $3.20 \pm 0.95 \%)(p>0.05)$.

\section{DISCUSSION}

\section{Rate of Nuclear Maturation}

Nuclear maturation is an essential prerequisite for the success of fertilization (Holubcová et al., 2019) and early embryonic development (Varghese et al., 2011). The results of our study revealed that nuclear maturation was improved by supplementation of the LC at a dose of $0.3 \mathrm{mg} / \mathrm{mL}$ in the maturation medium. These results are in line with the study in the oocytes of swamp buffalo (Phongmitr et al., 2013), mouse (Zare et al., 2015), and camel (Fathi \& El-Shahat, 2017) reporting that supplementation of LC in the medium of maturation could improve the nuclear maturation (MII stage) rate. The improvement of nuclear maturation may be related to the ability of LC to generate ATP (Agarwal et al., 2018), act as a ROS scavenger, and increase GSH levels, which are necessary for nuclear maturation (Phongmitr et al., 2013; Fathi \& El-Shahat. 2017).

The energy in the form of ATP is a critical point in the oocyte development for meiosis resumption (Leoni et al., 2015). Many researchers (Phongmitr et al., 2013; Zare et al., 2015; Fathi \& El-Shahat, 2017; Agarwal et al., 2018) state that LC plays an important role in lipid metabolism through its function as a cofactor for transporting fatty acids into the mitochondria through ß-oxidative processes to generate ATP production. Additionally, it has been shown that in the maturation process, the lipid content in the porcine oocytes has decreased, indicating that the utilization of lipid was the key energy source during oocyte maturation (Somfai et al., 2011; Phongmitr et al., 2013).

Table 2. Fertilization rate of sheep oocytes after treatment of L-carnitine in the different media

\begin{tabular}{|c|c|c|c|c|c|c|}
\hline \multirow{2}{*}{ Group } & \multicolumn{2}{|c|}{ LC treatment } & \multirow{2}{*}{ No of oocytes } & \multirow{2}{*}{$\begin{array}{l}\text { Total fertilization } \\
\text { rate }(\% \text {, mean } \pm \text { SD) }\end{array}$} & \multicolumn{2}{|c|}{ Pronuclear formation rate $(\%$, mean \pm SD) } \\
\hline & IVM & IVF & & & $2 \mathrm{PN}$ & $>2 \mathrm{PN}$ \\
\hline P0 & - & - & 103 & $70.9 \pm 2.2^{\mathrm{a}}$ & $62.1 \pm 2.5^{\mathrm{a}}$ & $8.8 \pm 3.9^{a}$ \\
\hline P1 & + & - & 101 & $80.2 \pm 2.8^{c}$ & $72.0 \pm 4.7^{\mathrm{b}}$ & $8.1 \pm 3.7^{a}$ \\
\hline $\mathrm{P} 2$ & - & + & 101 & $75.2 \pm 3.5^{\mathrm{b}}$ & $60.5 \pm 5.1^{\mathrm{a}}$ & $14.8 \pm 2.3^{\mathrm{b}}$ \\
\hline P3 & + & + & 98 & $80.4 \pm 3.4^{\mathrm{c}}$ & $68.2 \pm 2.7^{b}$ & $12.2 \pm 2.0^{\mathrm{ab}}$ \\
\hline
\end{tabular}

Note: $\mathrm{IVM}=$ in vitro maturation; $\mathrm{IVF}=$ in vitro fertilization; $2 \mathrm{PN}=2$ pronuclei; $>2 \mathrm{PN}=$ polyspermy (more than two pronuclei); $\mathrm{LC}=\mathrm{L}$-carnitine $(0.3 \mathrm{mg} /$ $\mathrm{mL}) ; \mathrm{P} 0=$ without LC supplementation in IVM and IVF medium as a control; P1= LC supplementation only in IVM medium; P2= LC supplementation only in IVF medium; P3= combination of LC supplementation in IVM and IVF media; Means in the same columns with different superscripts differ significantly $(\mathrm{p}<0.05)$. 


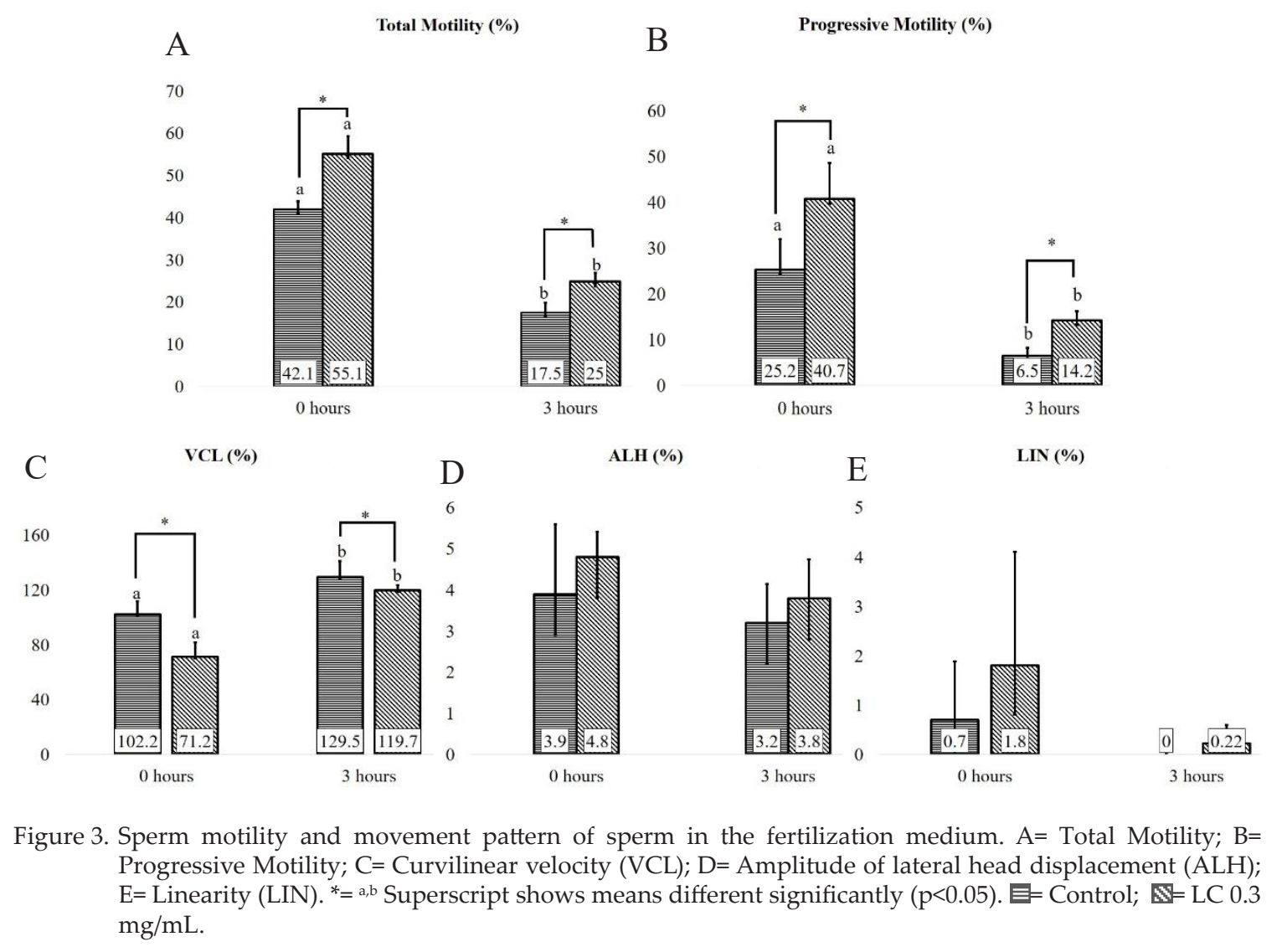

The ATP generating process was initiated by LC transfer into the tissue through the electrogenic force of the voltage-gated $\mathrm{Na}^{+}$-channels. The process utilizes organic cation transporter-2 (OCT2) to transport LC into the oocyte (Agarwal et al., 2018). In the oocytes, LC is converted into Acetyl-L-carnitine (ALC) by carnitine palmitoyl transferase-I (CPT-I) outside the membrane of mitochondria (Dunning \& Robker, 2017), and CPT-II promote the regeneration of carnitine from acyl-carnitine after translocation of long-chain fatty acids into the mitochondrial matrix. After conversion, LC will maintain the balance ratio of acetyl $\mathrm{CoA} / \mathrm{CoA}$ for maintaining glucose metabolism via the tricarboxylic acid cycle to produce higher energy (Agarwal et al., 2018).

On the other side, LC also has an antioxidant activity required to reduce ROS level in the oocytes during the in vitro maturation process (Wu et al., 2011; Fathi \& El-Shahat, 2017). Kelek et al. (2019) state that LC has a high antioxidant activity compared to the other antioxidants such as tocopherol and trolox. Furthermore, Zare et al. (2015) state that the antioxidant activity mechanism of LC may be done via a scavenging effect on radical anion, superoxide hydrogen peroxide, and 1,1-diphenyl2-picryl-hydrazyl free radical (DPPH). On the other side, it was reported that the level of apoptosis could be decreased after supplementation of LC in the maturation medium through its antioxidant action (Mansour et al., 2019).

Many researchers (Wu et al., 2011; Zare et al., 2015; Sovergino et al., 2017) state that LC can increase the level of GSH. In the oocyte nucleus, the accumulation of GSH has the potential to regulate the structure of chromatin and the dynamics of chromatin condensation (Vivancos et al., 2010). Those mechanisms are believed that supplementation of LC in the maturation medium improves the nuclear status of the oocyte by GSH generation.

Results of this study showed that supplementation of LC at a dose of $0.3 \mathrm{mg} / \mathrm{mL}$ had a higher nuclear maturation rate $(86.7 \%)$ compared to the LC supplementation at doses of 0.6 and $0.9 \mathrm{mg} / \mathrm{mL}(81.4 \%$ and $70.4 \%)$. It seems that a higher concentration of LC reduces the nuclear maturation rate. This result is similar to the result reported by Wu et al. (2011) that the higher concentration of LC causes a lower rate of maturation in the pig oocytes. Terada et al. (2002) reported that supplementation of antioxidants with improper concentrations would have toxic effects on the oocytes. Furthermore, Spinaci et al. (2019) reported that excessive supplementation of antioxidants, a dangerous condition called the antioxidant paradox, leading to oxidative stress that eventually reduced the percentage of nuclear maturation rate. In addition, Fathi \& El-Shahat (2017) reported that excessive LC concentrations would decrease lipid density that may not be beneficial for the further development of oocytes after fertilization.

\section{Cytoplasmic Maturation Rate and Pattern of Sperm Motility}

The success of in vitro fertilization would be optimal not only depend on the nuclear maturation but also on the cytoplasmic maturation (El-Raey \& Nagai, 2014; Riyuska et al., 2019). The cytoplasmic maturation of oocytes is required during fertilization and can be assessed based on the success of pronuclear formation (Kim et al., 2011; El-Raey \& Nagai, 2014). In the present study, 
it was revealed that supplementation LC at a dose of 0.3 $\mathrm{mg} / \mathrm{mL}$ only in the maturation medium (P1) and in the combination of the maturation and fertilization media (P3) showed that the formation of two pronuclear was higher than the other treatment and control. However, the levels of two pronuclear formations between those treatments were not significant. Therefore, it seems that the role of LC is greater in supporting pronuclear formation during the maturation process than fertilization. Therefore, supplementation of LC in the maturation medium is more effective to support pronuclear formation compared to the supplementation in the fertilization medium. The results found in our study are similar to the results of the study in camel (Fathi \& El-Shahat, 2017), reporting that the supplementation LC in the maturation medium can increase the in vitro fertilization rate.

The role of LC to support cytoplasmic maturation presumably through its effect on increasing the concentration of GSH after its supplementation in the maturation medium (Wu et al., 2011; Zare et al., 2015; Sovergino et al., 2017). The increasing concentration of GSH may be related to the role of LC as an antioxidant and thus can maintain GSH storages in the oocytes (Zare et al., 2015). On the other side, the increase in GSH concentration can be contributed by the role of ATP-dependent enzymes in the synthesis of GSH (Sovergino et al., 2017). In $\beta$-oxidation of long-chain fatty acids, 1-carnitine plays a critical role in generating ATP by providing a system of transmission for free fatty acids into the mitochondria (Jeulin \& Lewin. 1996). Therefore, the process of $\beta$-oxidation is required by the oocytes to support nuclear and cytoplasmic maturation (Dunning et al., 2010). Furthermore, our finding strongly suggested that the formation of two pronuclear in the treatment group of LC supplementation in the fertilization medium only (P2) was lower than that in the LC supplementation in the maturation medium only (P1). This result indicates that the role of LC is more beneficial when it is supplemented in the IVM medium compared to in the IVF medium to support the cytoplasmic maturation.

Our additional finding revealed that supplementation of LC in the fertilization medium only (P2) could increase polyspermy (14.8\%) compared to the other treatments, which has an impact on the failure of early embryonic development. The incidence of polyspermy in IVEP systems is not yet clearly understood. However, it might be caused by various factors, either from the spermatozoa or oocytes (Goncalves et al., 2010). Furthermore, Itoi et al. (2018) suggest that a higher number of motile sperms results in more frequent contact with an ovum, thus increasing the likelihood of polyspermy. Therefore, an assessment pattern of sperm motility was carried out in this study to observe total motility, progressive motility, VCL, ALH, and LIN.

The results showed that the supplementation of LC in the fertilization medium significantly increased the total motility, progressive motility, and hyperactivity assessed by increasing the percentage of curvilinear velocity (VCL) compared to controls. Those results may be the factors that could be the cause of increasing the incidence of polyspermy in the treatment group of LC supplementation in the fertilization medium only (P2). Agarwal et al. (2018) suggested that the increased sperm motility and hyperactivity could be related to the role of LC in providing energy in the form of ATP used by the sperms.

\section{CONCLUSION}

L-carnitine supplementation at the concentration of $0.3 \mathrm{mg} / \mathrm{mL}$ in the maturation medium can improve nuclear maturation, therefore support a better cytoplasmic maturation, which is characterized by the high fertilization rate. The further finding indicates that LC supplementation in the maturation medium only can support a better fertilization rate, which is presumably related to GSH production during maturation as a prerequisite for pronuclear formation. Meanwhile, total motility, progressive motility, and curvilinear velocity (VCL) increase in the fertilization medium containing LC, but it does not significantly improve the fertilization rate.

\section{CONFLICT OF INTEREST}

The authors declare that there is no conflict of interest that could be perceived as prejudicing the impartiality of the research reported.

\section{ACKNOWLEDGEMENT}

This study was supported by Pendanaan Penelitian, Skema Tesis Magister T.A. 2020, the Ministry of Research and Technology/National Agency for Research and Innovation, Republic of Indonesia.

\section{REFERENCES}

Agarwal, A., P. Sengupta, \& D. Durairajanayagam. 2018. Role of L-carnitne in female infertility. Reprod. Biol. Endocrinol. 16:2-18. https://doi.org/10.1186/s12958-018-0323-4

Curnow, E. C., J. P. Ryan, D. M. Saunders, \& E. S. Hayes. 2010. Oocytes glutathion and fertilization outcome of Macaca nemestrina and Macaca fascicularis in in vivo- and in vitromatured oocytes. Reprod. Fertil. Dev. 22:1032-1040. https:// doi.org/10.1071/RD09308

Dokmeci, D. 2005. Oxidative stress, male infertility and the role of carnitines. Folia Med. 47:26-30.

Dunning, K. R., K. Cashman, D. L. Russell, J. G. Thompson, R. J. Norman, \& R. L. Robker. 2010. Beta-oxidation is essential for mouse oocyte developmental competence and early embryo development. Biol. Reprod. 83:909-918. https:// doi.org/10.1095/biolreprod.110.084145

Dunning, K. R. \& R. L. Robker. 2017. The role of 1-carnitine during oocyte in vitro maturation: Essential co-factor. Anim. Reprod. 14:469-475. https://doi. org/10.21451/1984-3143-AR988

Egashira, J., Y. Iraha, H. Khatun, Y. Wada, T. Konno, H. Tatemoto, \& K. Yamanaka. 2019. Efficient in vitro embryo production using in vivo-matured oocytes from superstimulated Japanese black cows. J. Reprod. Dev. 65:183-190. https://doi.org/10.1262/jrd.2018-155

El-Raey, M. \& T. Nagai. 2014. Different aspect of cattle in vitro maturation. J. Reprod. Infertil. 5:1-13.

Fathi, M. \& K. H. El-Shahat. 2017. L-carnitine enhance oocyte maturation and improves in vitro development of 
embryos in dromedary camels (Camelus dromedaries). Theriogenology. 104:18-22. https://doi.org/10.1016/j. theriogenology.2017.08.006

Goncalves. F. S, L. S. S. Barretto, R. P. Arruda, S. H. V. Perri, \& G. Z. Mingoti. 2010. Effect of antioxidants during bovine in vitro fertilization procedures on spermatozoa and embryo development. Reprod. Dom. Anim. 45:129-135. https://doi.org/10.1111/j.1439-0531.2008.01272.x

Holubcová, Z., D. Kyjovská, M. Martonová, D. Páralová, T. Klenková, P. Otevřel, R. Štěpánová, S. Kloudová, \& A. Hampl. 2019. Egg maturity assessment prior to ICSI prevents premature fertilization of late maturing oocytes. J. Assist. Reprod. Genet. 36:445-452. https://doi.org/10.1007/ s10815-018-1393-0

Itoi, F., Y. Tamaki, S. Ohnishi, E. Araki, Y. Ogasawara, K. Aihara, H. Sato, K. Nishikawa, M. Ohmoto, Y. Otani, H. Sakaguchi, \& T. Isobe. 2018. A study on the relationship between the fertilization rate in IVF and the mechanical energy of sperm. J. Pregnancy Reprod. 2:1-4. https://doi. org/10.15761/JPR.1000142

Jiang, W, Y. Li, Y. Zhao, Q. Gao, Q. Jin, C. Yan, \& Y. Xu. 2020. L-carnitine supplementation during in vitro culture regulates oxidative stress in embryos from bovine aged oocytes. Theriogenology. 143:64-73. https://doi.org/10.1016/j. theriogenology.2019.11.036

Jeulin, C. \& L. M. Lewin. 1996. Role of free L-carnitine and acetyl-L-carnitine in post-gonadal maturation of mammalian spermatozoa. Hum. Reprod. Update. 2:87-102. https://doi. org/10.1093/humupd/2.2.87

Kelek, S. E., E. Afşar, G. Akcay, B. Danisman, \& M. Aslan. 2019. Effect of chronic l-carnitine supplementation on carnitine levels, oxidative stress and apoptitic markers in peripheral organs of adult wistar rats. Food Chem. Toxicol. 134:1-7. https://doi.org/10.1016/j.fct.2019.110851

Leoni, G. G., M. G. Palmerini, V. Satta, S. Succu, V. Pasciu, A. Zinellu, C. Carru, G. Macchiarelli, S. A. Nottola, S. Naitana, \& F. Berlinguer. 2015. Differences in the kinetic of the first meiotic division and in active mitochondrial distribution between prepubertal and adult oocytes mirror differences in their developmental competence in a sheep model. PloS One. 10:e0124911. https://doi.org/10.1371/ journal.pone.0124911

Lobo, V., A. Pati, A. Phatak, \& N. Chandra. 2010. Free radicals, antioxidants and functional food: Impact on human health. Pharmacogn. Rev. 4:118-126. https://doi. org/10.4103/0973-7847.70902

Lopes, A. S., M. Lane, \& J. G. Thompson. 2010. Oxygen consumption and ros production are increased at the time of fertilization and cell cleavage in bovine zygotes. Hum. Reprod. 25:2762-2773. https://doi.org/10.1093/humrep/ deq221

Mansour, G.H., H. Abdelrazik, R. K. Sharma, E. Radwan, T. Falcone, \& A. Agarwal. 2019. L-carnitine supplementation reduces oocyte cytoskeleton damage and embryo apoptosis induced by incubation in peritoneal fluid form patients with endometriosis. Fertil. Steril. 91:2079-2086. https://doi. org/10.1016/j.fertnstert.2008.02.097

Opuwari, C. S \& R. R. Henkel. 2016. An update on oxidative damage to spermatozoa and oocytes. Biomed. Res. Int. 2016:9540142. https://doi.org/10.1155/2016/9540142

Paramio, MT., \& D. Izquierdo. 2014. Current status of in vitro embryo production in sheep and goats. Reprod. Dom. Anim. 49:37-48. https://doi.org/10.1111/rda.12334

Phongmitr, T., Y. Liang, K. Srirattana, K. Panyawai, N. Sripunya, C. Treetampinich, \& R. Parnpai. 2013. Effects of L-carnitine supplemented in maturation medium on the maturation rate of swamp buffalo oocytes. Buffalo Bull. 32:613-616.

Reader, K. L., J. Stanton, \& J. L. Juengel. 2017. The role of oocyte organelles in determining developmental competence.
Biology. 6:1-22. https://doi.org/10.3390/biology6030035

Riyuska, A., N. W. K. Karja, \& M. A. Setiadi. 2019. Efficacy of leptin supplementation on nuclear maturation and fertilization rate of sheep oocyte. Trop. Anim. Sci. J. 42:1-5. https://doi.org/10.5398/tasj.2019.42.1.1

Shafiei, G., M. Almasi, H. Nikzad, J. Miyan, J. A. Mahabadi, \& G. Moshkdanian. 2020. L-carnitine reduces the adverse effect of ROS and up-regulates the expression of implantation related genes in in vitro developed mouse embryos. Theriogenology. 145:59-66. https://doi.org/10.1016/j. theriogenology.2020.01.008

Shirazi, A. \& Sadeghi, N. 2007. The effect of ovine oocyte diameter on nuclear maturation. Small Rumin. Res. 69:103-107. https://doi.org/10.1016/j.smallrumres.2005.12.022

Somfai, T., M. Kaneda, S. Akagi, S. Watanabe, S. Haraguchi, E. Mizutani, \& T. Q. D. Nguyen. 2011. Enhancement of lipid metabolism with L-carnitine during in vitro maturation improves nuclear maturation cleavage ability of follicular porcine oocytes. Reprod. Fertil. Dev. 23:912-920. https:// doi.org/10.1071/RD10339

Sovergino, T.C., P. R. Adona, P. S. Monzani, S. Guemra, F. D. A. Barros, F. G. Lopes, \& C. L. V. Leal. 2017. Effects of supplementation of medium with different antioxidant during in vitro maturation of bovine oocyte on subsequent embryo production. Reprod. Domest. Anim. 52:561-569. https://doi.org/10.1111/rda.12946

Spinaci, M., D. Bucci, V. Muccilli, N. Cardullo, C. Nerozzi, G. Galeati. 2019. A polyphenol-rich extract from an oenologicaloak-derived tannin influences in vitro maturation of porcine oocytes. Theriogenology. 129:82-89. https://doi. org/10.1016/j.theriogenology.2019.02.017

Suzuki, K., B. Eriksson, H. Shimizu, T. Nagai, \& H. RodriguezMartinez. 2000. Effect of hyaluronan on monospermic penetration of porcine oocytes fertilized in vitro. Int. J. Androl. 23:13-21. https://doi.org/10.1046/j.1365-2605.2000. t01-1-00198.x

Terada, S., T. Nishiramura, M. Sasaki, H. Yamada, \& M. Miki. 2002. Sericin, a protein derived from silkworms, accelerates the proliferation of several mammalian cell lines including hybridoma. Cytotechnology. 40:3-12. https://doi. org/10.1023/A:1023993400608

Troung, T. \& D. K. Gerdner. 2017. Antioxidants improve IVF outcome and subsequent embryo development in the mouse. Hum. Reprod. 32:2404-2413. https://doi. org/10.1093/humrep/dex330

Varghese, A. C., K. D. Ly, C. Corbin, J, Mendiola, \& A. Agarwal. 2011. Oocyte developmental competence and embryo development: Impact of lifestyle and environmental risk factors. Reprod. Biomed. Online. 22:410-420. https://doi. org/10.1016/j.rbmo.2010.11.009

Vivancos, P. D., T. Wolff, J. Markovic, F. V. Pallardo, \& C. H. Foyer. 2010. A nuclear glutathione cycle within the cell cycle. Biochem. J. 431:169-178. https://doi.org/10.1042/ BJ20100409

Wang, S., G. He, M. Chen, T. Zou, W. Xu, \& X. Liu. 2017. The role of antioxidant enzymes in the ovaries. Oxid. Med. Cell. Longev. 2017:4371714. https://doi. org/10.1155/2017/4371714

Wu, G. Q., B. Y. Jia, J. J. Li, X. W. Fu, B. G. Zhou, Y. P. Hou, \& S. E. Zhu. 2011. L-carnitine enhances oocyte maturation and development of parthenogenetic embryos in pigs. Theriogenology. 76:785-793. https://doi.org/10.1016/j. theriogenology.2011.04.011

Zare, Z., R. M. Farahani, M. Salehi, A. Priryaei, M. G. Novin, F. F. Fathabadi, M. Mohammadi, \& M. DehghaniMohammadabadi. 2015. Effect of L-carnitine on maturation and early embryo development of immature mouse oocytes selected by brilliant cresyle bule staining. J. Assist. Reprod. Genet. 32:635-643. https://doi.org/10.1007/ s10815-015-0430-5 